Check for updates

Cite this: Phys. Chem. Chem. Phys., 2022, 24, 5669

Received 12th November 2021 Accepted 2nd February 2022

DOI: $10.1039 / d 1 c p 05187 c$

rsc.li/pccp

\title{
The photodissociation of solvated cyclopropanone and its hydrate explored via non-adiabatic molecular dynamics using $\triangle \mathrm{SCF}$
}

\author{
Eva Vandaele, (D) Momir Mališ and Sandra Luber (D) *
}

\begin{abstract}
The decay of cyclopropanone is a typical example of a photodecomposition process. Ethylene and carbon monoxide are formed following the excitation to the first singlet excited state through a symmetrical or asymmetrical pathway. The results obtained with non-adiabatic molecular dynamics (NAMD) using the delta self-consistent field ( $\triangle S C F$ ) method correspond well to previous experimental and multireference theoretical studies carried out in the gas phase. Moreover, this efficient methodology allows NAMD simulations of cyclopropanone in aqueous solution to be performed, which reveal analogue deactivation mechanisms, but a shorter lifetime and reduced photodissociation as compared to the gas-phase. The excited state dynamics of cyclopropanone hydrate, an enzyme inhibitor, in an aqueous environment are reported as well. Cyclopropanone hydrate strongly interacts with the surrounding solvent via the formation of hydrogen bonds. Excitation to the first singlet excited state shows an asymmetric pathway with cyclopropanone hydrate and propionic acid as the main photoproducts.
\end{abstract}

\section{Introduction}

Cyclopropanone is the smallest cyclic ketone and is known for its high reactivity. ${ }^{1}$ In fact, it was first studied as a reaction intermediate, ${ }^{2-4}$ e.g. in the Favorskii reaction, ${ }^{5}$ before a synthetic method was developed. ${ }^{6,7}$ Cyclopropanone can be examined at low temperatures or after the addition of polymerisation inhibitors. It is also formed from ethylene and carbon monoxide in interstellar ice, where astrophysicists identified it as a tracer for rapid low temperature non-equilibrium synthesis. ${ }^{8}$ An experimental study of the gas-phase photochemistry yielded ethylene, carbon monoxide and polymers as photoproducts. ${ }^{9}$ However, polycyclopropanone was formed in negligible amounts and was ascribed to the thermal reaction. Fluorescence was observed only at wavelengths above $365 \mathrm{~nm} .^{10,11}$ Based on the rapid photolysis with a reaction time of $\leq 200 \mathrm{ps}$, a predissociation process to a biradical was proposed, in contrast to the intersystem crossing mechanism of other cyclic ketones. ${ }^{9,12}$ The excitation to the ${ }^{1}\left(n \rightarrow \pi^{*}\right)$ state weakens the lateral $\mathrm{C}-\mathrm{C}$ bond (i.e. the $\mathrm{C}-\mathrm{C}$ bond adjacent to the carbonyl group) strength of the already strained cyclopropanone resulting in the asymmetric dissociation.

After the first few experimental studies, several types of computational investigations on the photodissociation of a

Department of Chemistry, University of Zürich, Winterthurerstrasse 190, 8057

Zürich, Switzerland.E-mail: sandra.luber@chem.uzh.ch cyclopropanone molecule were performed as well. ${ }^{9-11,13-15}$ A minimum-energy conical intersection (MECI) near the first singlet excited state $\left(\mathrm{S}_{1}\right)$ minimum promoting ultrafast internal conversion was identified with complete active space selfconsistent field (CASSCF) calculations. ${ }^{10}$ Upon excitation to the $S_{1}$ state, a fast non-radiative relaxation process, internal conversion and dissociation in the ground state were observed using non-adiabatic molecular dynamics (NAMD). The $\mathrm{S}_{1}$ minimum and CI geometries exhibit an out-of-plane oxygen position. A successive state-averaged CASSCF study identified a second MECI and corresponding decarbonylation mechanism. ${ }^{11}$ The second pathway is comprised of a symmetrical structure where the lateral $\mathrm{C}-\mathrm{C}$ bonds are broken simultaneously. Whereas the multistate multireference complete active space second-order perturbation (MS-MR-CASPT2) method provided a lower energy for the asymmetrical CI, ensemble density functional theory (DFT) returned an insignificant energy difference. ${ }^{13}$ Nevertheless, both methods found that the majority of the trajectories decay through the former mechanism in the surface hopping calculations. Hopping events were found to fit a bi-Gaussian curve, while the $S_{1}$ population follows a biexponential decay function with lifetimes of $33 \mathrm{fs}$ and $95 \mathrm{fs}^{13}$

Once the existence and molecular structure of cyclopropanone were confirmed in the mid-1960s, the reactivity with a range of electrophiles and nucleophiles in various media was studied. ${ }^{16-18}$ The first experiments in aqueous environment generated an adduct without ring opening at the low yield of 
approximately $30 \% .^{19}$ Later studies found that the 1,1dihydroxycyclopropane is stable in water for several days, after which propionic acid is slowly formed. ${ }^{2,20}$ Biochemists use cyclopropanone hydrate to design enzyme inhibitors. ${ }^{21-24}$ The hydrate yield depends on the water-cyclopropanone ratio. Purification of the hydrate at room temperature, heating or basic conditions result in isomerisation to propionic acid. ${ }^{2,21,25}$ Polymerisation of the hydrate into dihydroxy ether was observed using traces of water in the presence of methylene chloride. $^{25}$ To the best of our knowledge, no quantitative data on the equilibrium constant between cyclopropanone and cyclopropanone hydrate in an aqueous environment is available. Therefore, the photodynamics of both the hydrate and cyclopropanone in water were simulated in this work.

To date, only cyclopropanone in a vacuum has been explored by means of NAMD. Quantum mechanics/molecular mechanics (QM/MM) is the most popular method to perform NAMD calculations of molecules in solution. ${ }^{26-28}$ Nevertheless, as the photodynamics is unknown, the prior partitioning of the system and the deficient description of polarisation effects could result in inadequate conclusions regarding the photodissociation process of cyclopropanone and its hydrate in an aqueous environment. $^{29}$ The delta self-consistent field $(\triangle \mathrm{SCF})$ method has been successfully applied to examine surface hopping dynamics in solvated systems with full atomistic DFT simulations, using a mixed Gaussian and plane wave approach. ${ }^{30,31}$ The method can be a more accurate alternative to QM/MM, while still computationally efficient, for the description of liquids or other systems obeying periodic boundary conditions.

\section{Methods}

\subsection{Theory}

NAMD is a popular method to investigate the time evolution of electronic populations after photoexcitation. ${ }^{32,33}$ It is a non-BornOppenheimer, mixed quantum-classical approach, in which the nuclei are propagated classically based on the quantum mechanically calculated electronic structure. More specifically, the nuclear coordinates are updated using the gradients of the occupied electronic state. Non-adiabatic transitions between electronic states are stochastic and instantaneous. ${ }^{34}$ A multitude of independent trajectories are propagated to derive statistically relevant conclusions. Of the various NAMD algorithms and formulations, Tully's fewest-switches surface-hopping (TFSSH) ${ }^{35}$ was used in this work.

The time evolution of the electronic wavefunction $|\Psi(r ; t ; \mathbf{R})\rangle$ is obtained by expansion in a linear combination of adiabatic electronic eigenfunctions $\left|\psi_{i}\left(\mathbf{R}_{t}\right)\right\rangle$ corresponding to electronic state $i$ with energy $E_{i}$

$$
\begin{aligned}
& \left|\Psi\left(\mathbf{R}_{t}\right)\right\rangle=\sum_{i} \phi_{i}\left(\mathbf{R}_{t}\right)\left|\psi_{i}\left(\mathbf{R}_{t}\right)\right\rangle, \\
& \hat{H}_{\mathrm{e}}\left(\mathbf{R}_{t}\right)\left|\psi_{i}\left(\mathbf{R}_{t}\right)\right\rangle=E_{i}\left(\mathbf{R}_{t}\right)\left|\psi_{i}\left(\mathbf{R}_{t}\right)\right\rangle,
\end{aligned}
$$

where $\mathbf{R}_{t}$ is the nuclear coordinates at time $t$ and $\hat{H}_{\mathrm{e}}\left(\mathbf{R}_{t}\right)$ is the electronic Hamiltonian, while the electron coordinates $\mathbf{r}$ are omitted for simplicity. The population of the electronic state $i$ equals the square of the absolute value of the corresponding coefficient $\phi_{i}\left(\mathbf{R}_{t}\right)$. As a result, the evolution of the molecular system can be calculated by integrating the time-dependent Schrödinger equation (TDSE) for the state coefficients

$$
\begin{aligned}
i \hbar \frac{\partial \phi_{i}\left(\mathbf{R}_{t}\right)}{\partial t}= & E_{i}\left(\mathbf{R}_{t}\right) \phi_{i}\left(\mathbf{R}_{t}\right) \\
& -i \hbar \sum_{i \neq j} \phi_{j}\left(\mathbf{R}_{t}\right)\left\langle\psi_{i}\left(\mathbf{R}_{t}\right)\left|\frac{\partial}{\partial t}\right| \psi_{j}\left(\mathbf{R}_{t}\right)\right\rangle .
\end{aligned}
$$

The bracket in the second right-hand-side term of eqn (3), called the first-order non-adiabatic coupling (NAC), $D_{i j}^{\mathrm{NAC}}\left(\mathbf{R}_{t}\right)$, enables the transfer of population between states. The TDSE is integrated in infinitesimal steps. At each step, the hopping probability $\left(P_{i \rightarrow j}\right)$ is calculated and compared to a random number $(\xi)$ generated from a uniform distribution. If a hop is accepted $\left(P_{i \rightarrow j}>\xi\right)$, all velocities are rescaled to guarantee the conservation of energy, by distributing the additional kinetic energy over all atoms in the box. In a case where the molecule's kinetic energy is insufficient, the transition is rejected. A decoherence correction is also applied at every step to damp the overpopulated state coefficients. ${ }^{36}$ The adiabatic TFSSH hopping probability with nuclear time step $\Delta t$ is equal to ${ }^{37}$

$$
P_{i \rightarrow j}^{\mathrm{TFSSH}}=\max \left[0, \frac{2 \Delta t}{\left|\phi_{i}\left(\mathbf{R}_{t}\right)\right|^{2}} \operatorname{Re}\left(D_{i j}^{\mathrm{NAC}}\left(\mathbf{R}_{t}\right) \phi_{i}^{*}\left(\mathbf{R}_{t}\right) \phi_{j}\left(\mathbf{R}_{t}\right)\right)\right] .
$$

\section{$2.2 \Delta \mathrm{SCF}$}

In NAMD, the trajectories are propagated on-the-fly. To be more precise, the state coefficients and nuclear coordinates are evolved simultaneously based on the state energies, gradients and NACs calculated at each integration step. Hence, the accuracy of the dynamics is determined by the underlying electronic structure theory. The DFT-based $\triangle \mathrm{SCF}$ method is a variational method that independently optimizes excited state electronic densities $\left(\rho_{i}(\vec{r})\right.$ corresponding to electronic state $\left.i\right){ }^{38-45}$ Excited electronic states of systems with discrete eigenstates are obtained by a Kohn-Sham (KS) DFT optimisation with nonAufbau fixed KS molecular orbital (MO) occupations $\left(n_{j}^{i}\right.$ corresponding to KS MO $\left.\varphi_{j}^{i}(\vec{r})\right)$ using the ground-state algorithms, so that the total final electron density resembles that of a certain excited electronic state $i$.

$$
\rho_{i}(\vec{r})=\sum_{j} n_{j}^{i}\left|\varphi_{j}^{i}(\vec{r})\right|^{2}
$$

The sum of the orbital occupation numbers equals the total number of electrons $N_{\mathrm{e}}\left(\sum_{j} n_{j}^{i}=N_{\mathrm{e}}\right)$ and each KS MO $\varphi_{j}^{i}(\vec{r})$ is an eigenfunction of the KS equation corresponding to eigenenergy $\varepsilon_{j}^{i}$

$$
\left(-\frac{\hbar^{2}}{2} \nabla^{2}+w(\vec{r})+\int \frac{\rho_{i}\left(\vec{r}^{\prime}\right)}{\left|\vec{r}-\vec{r}^{\prime}\right|} \mathrm{d} \vec{r}^{\prime}+v_{\mathrm{xc}}\left[\rho_{i}\right](\vec{r})\right) \varphi_{j}^{i}(\vec{r})=\varepsilon_{j}^{i} \varphi_{j}^{i}(\vec{r}) .
$$

The kinetic energy operator for non-interacting electrons, the external electrostatic interaction, electron-electron 
repulsion and exchange-correlation potentials are summed in the left-hand side respectively.

To reproduce the desired electronic state multiplicity, the individual electron spin densities must also match the spin properties of the targeted excited electronic state. In the case of singlet excited electronic states the alpha and beta spin densities must be equal. This can be assured by using the restricted KS formulation, ${ }^{43,46,47}$ while by using rounded MO occupation numbers, a single Slater determinant is adequate to approximate the wave function of a single reference excited electronic state. ${ }^{30}$ MO occupation numbers for the excited state, either fractional or rounded, have to be provided, for example using time-dependent DFT (TDDFT). ${ }^{30} \Delta \mathrm{SCF}$ has provided good results for a variety of systems, ${ }^{48-50}$ thereby showing an improved performance as compared to TDDFT e.g. for the description of low-lying charge transfer states, ${ }^{51,52}$ and for NAMD studies in the liquid phase. ${ }^{30,31,44}$

\subsection{Transition dipole moment}

As the electronic states optimised by $\triangle \mathrm{SCF}$ are not mutually orthogonal, a procedure to expand the excited state's approximated Slater determinant into a linear combination of singlyexcited Slater determinants constructed from ground state KS MOs has been recently developed in our group. ${ }^{53}$ This alleviates the non-orthogonality between the ground and excited electronic $\triangle$ SCF states. The transition dipole moment (TDM) operator $\hat{\boldsymbol{\mu}}$ between the ground and singlet excited state $i$ is given by

$$
\left\langle\psi_{\mathrm{S}_{0}}|\hat{\boldsymbol{\mu}}| \psi_{\mathrm{S}_{i}}\right\rangle=\sqrt{2} \sum_{\mathrm{ov}} C_{\mathrm{ov}}^{\mathrm{S}_{i}}\left\langle\varphi_{\mathrm{o}}^{\mathrm{S}_{0}}|\hat{\boldsymbol{\mu}}| \varphi_{\mathrm{v}}^{\mathrm{S}_{0}}\right\rangle
$$

with $C_{\mathrm{ov}}^{\mathrm{S}_{i}}$ being the expansion coefficients from the projection of the singlet $\triangle \mathrm{SCF}$ excited electronic state Slater determinant $\psi_{\mathrm{S}_{i}}$ onto the singly-excited Slater determinants constructed by promoting one electron from the ground state KS occupied $(o) \mathrm{MO} \varphi_{\mathrm{o}}^{\mathrm{S}_{0}}$ to a virtual $(v) \mathrm{MO} \varphi_{\mathrm{v}}^{\mathrm{S}_{0}}$,

$$
C_{\mathrm{ov}}^{\mathrm{S}_{i}}=\left\langle\psi_{\mathrm{o} \alpha \rightarrow \mathrm{v} \alpha}^{\mathrm{S}_{0}} \mid \psi_{\mathrm{S}_{i}}\right\rangle+\left\langle\psi_{\mathrm{o} \beta \rightarrow \mathrm{v} \beta}^{\mathrm{S}_{0}} \mid \psi_{\mathrm{S}_{i}}\right\rangle .
$$

Depending on the exact dipole moment operator $\hat{\boldsymbol{\mu}}$, the oscillator strength of the excitation in the length representation

$$
\begin{aligned}
& \left(\hat{\boldsymbol{\mu}}_{L}=\sum_{j}^{N_{\mathrm{e}}} \hat{\mathbf{r}}_{j}, \text { in atomic units }\right) \text { then is } \\
& \qquad f_{\mathrm{S}_{0} \rightarrow \mathrm{S}_{i}}^{L}=\frac{2}{3}\left\|\left\langle\psi_{\mathrm{S}_{0}}\left|\hat{\boldsymbol{\mu}}_{L}\right| \psi_{\mathrm{S}_{i}}\right\rangle\right\|^{2}\left(E_{\mathrm{S}_{i}}-E_{\mathrm{S}_{0}}\right),
\end{aligned}
$$

or in the velocity representation $\left(\hat{\boldsymbol{\mu}}_{V}=l \sum_{j}^{N_{\mathrm{e}}} \partial / \partial \boldsymbol{r}_{j}\right.$, in atomic units)

$$
f_{\mathrm{S}_{0} \rightarrow \mathrm{S}_{i}}^{V}=\frac{2\left\|\left\langle\psi_{\mathrm{S}_{0}}\left|\hat{\boldsymbol{\mu}}_{V}\right| \psi_{\mathrm{S}_{i}}\right\rangle\right\|^{2}}{3\left(E_{\mathrm{S}_{i}}-E_{\mathrm{S}_{0}}\right)} .
$$

The procedure can be applied to calculate various observables, including the spin-orbit coupling between singlet and triplet states. ${ }^{53}$

\subsection{Computational settings}

The NAMD calculations were performed by the Zagreb surface hopping code ${ }^{54}$ interfaced with a modified version of the CP2K program package. ${ }^{55}$ A new interface script was written in Python to couple the codes in a simpler and versatile manner. The interface script provides the input geometry and setup to the $\mathrm{CP} 2 \mathrm{~K}$ code, and reads the energies, nuclear gradients and KS MO coefficients. It calculates the NAC terms using the TullyHammes-Schiffer approximation ${ }^{56}$ and provides all the required components to the NAMD code. The same Python script was used for the calculation of absorption spectra with expressions (9) and (10). Phases between KS MOs of consecutive steps are adjusted to maintain the NAC continuity. All DFT and $\triangle \mathrm{SCF}$ electronic structure calculations were performed with CP2K.

Initial gas-phase configurations were sampled from the thermalised Wigner distribution at $300 \mathrm{~K}$, based on the projected Hessian obtained at a ground electronic state optimised geometry at the BLYP/TZV2P-GTH level of theory. ${ }^{57,58}$ Since the mixed Gaussian and plane wave method for DFT as implemented in the CP2K programme was used, the cyclopropanone molecule was confined at the center of a non-periodic cubic box with a side length of $15 \AA$.

Initial configurations for the condensed phase were sampled from DFT-MD simulations. The solutes were centered in a pre-equilibrated box containing 32 water molecules at ambient conditions, thereby replacing 7 water molecules. The cubic box was relaxed using the isotropic isothermal-isobaric (NPT) ensemble at $300 \mathrm{~K}$ and 1 bar, using corresponding NoseHoover algorithms with time constants of $20 \mathrm{fs}$ and $200 \mathrm{fs}$ for the thermostat and barostat respectively and a 0.5 fs timestep at the BLYP/TZV2P-GTH level of theory. The 250 final initial phase space points were evenly spaced sampled from a 45 ps long trajectory using the canonical ensemble (NVT) at $300 \mathrm{~K}$ with a fixed cell box length of $9.26 \AA$ and $9.12 \AA$ for the solvated cyclopropanone and cyclopropanone hydrate respectively.

The UV absorption spectrum was constructed from the initial conformations, as sampled from the Wigner distribution or DFT-MD NVT trajectories, by calculating the wavelengths and corresponding oscillator strengths of the first singlet excitation at the BLYP/TZV2P-GTH level of theory. The spectra were broadened using a Lorentzian function with a half-width at half-maximum height of $10 \mathrm{~nm}$.

The TFSSH algorithm, with a 0.5 fs timestep, was used for all trajectories which were propagated for 500 steps. For each system, 250 NAMD trajectories were propagated. $\triangle$ SCF single point calculations were performed at the B3LYP/TZV2P-GTH level of theory using the Goedecker-Teter-Hutter pseudopotentials and Grimme D3 dispersion correction without damping. ${ }^{59,60}$ These settings lead to good agreement with experimental data in previous hybrid Gaussian plane wave simulations of liquid water. $^{61,62}$ The Coulomb potential was truncated at $10 \AA$ and a 500 Ry cutoff for the plane wave basis was set.

In analogy to previous studies, only the ground and first singlet excited state populations were evolved in the NAMD. ${ }^{10,11,13-15}$ The optimised ground state MOs served as an initial guess for the optimisation of the excited state. The first singlet excited state was obtained by promoting an electron 
from the highest occupied MO (HOMO) to the lowest unoccupied MO (LUMO). By default, the SCF was performed using the standard diagonalisation method in $\mathrm{CP} 2 \mathrm{~K},{ }^{63}$ where density mixing was employed for each SCF iteration and the mixing fraction of the new density was reduced from 0.4 to 0.1 after 25 steps. The interface script calling $\mathrm{CP} 2 \mathrm{~K}$ on the fly contained an SCF convergence procedure, which was automatically applied in case the default SCF energy minimisation could not converge. ${ }^{30}$ In the case of energy oscillations, the fraction of new density included in the density mixing procedure was further reduced. In the case of convergence problems, a level shift of 0.1 Hartree during the SCF convergence, the orbital transformation method and the self-consistent subspace refinement by diagonalisation of the Hamiltonian were consecutively tried. The trajectory was discarded if no method converged. Trajectories with cumbersome excited state convergence after deexcitation to the ground electronic state were further propagated using DFT-MD.

The MECI structures were obtained at the $\triangle$ SCF level with the aid of the CIOpt programme. ${ }^{64}$ TDDFT calculations were performed in Turbomole, ${ }^{65}$ while the state-averaged CASSCF results were obtained using Gaussian ${ }^{66}$ and the extended multistate complete active space second-order perturbation theory (XMS-CASPT2) results were calculated using the BAGEL programme. ${ }^{67}$ For the latter two methods, an active space consisting of eight electrons in the $\mathrm{C}=\mathrm{O} \pi$ and $\pi^{*}$ orbitals, the $\sigma$ and $\sigma^{*}$ orbitals of both lateral C-C bonds, and one nonbonding $n$ orbital on the oxygen atom has been used, in analogy to previous cyclopropanone studies. ${ }^{14,15}$ The def2-TZVPP basis set $^{68}$ was used with Turbomole and Gaussian, while TZVPP ${ }^{69}$ was used with BAGEL. The XMS-CASPT2 based NAMD studies were performed by coupling the BAGEL electronic structure programme ${ }^{67,70-72}$ to the Zagreb surface hopping code. ${ }^{54}$

\section{Results and discussion}

\subsection{Cyclopropanone in a vacuum}

The UV absorption spectrum of isolated cyclopropanone was calculated from the 250 sampled NAMD initial configurations using the procedure for calculating the TDM detailed above. Its absorption spectra in both the length and the velocity representation are very similar to the experimental and theoretical spectra in ref. 13. The maxima are red-shifted by $7 \mathrm{~nm}$ and $15 \mathrm{~nm}$ for the length and velocity representations respectively as compared to the experimental value of $312 \mathrm{~nm}$, as indicated in Fig. 1. Hence, the initial conditions sampled for the NAMD form a good representation for the distribution of the cyclopropanone excitation energies.

The optimised geometries and corresponding frontier orbitals at the minima and MECIs as obtained with the B3LYP/ TZV2P-GTH $\triangle$ SCF method are depicted in Fig. 2. The geometrical parameters at the $\mathrm{S}_{0}$ minimum correspond well to experimental values obtained from microwave spectral data, ${ }^{73}$ as listed in Table 1 . The optimised $\mathrm{S}_{1}$ minimum geometry is characterised by slightly elongated lateral $\mathrm{C}-\mathrm{C}$
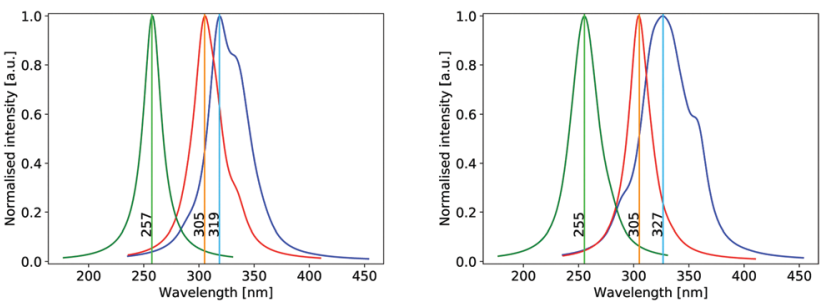

Fig. 1 Normalised theoretical absorption spectra of cyclopropanone (blue), solvated cyclopropanone (red) and cyclopropanone hydrate (green) obtained with $\triangle \mathrm{SCF}$ using the electric dipole operator in the length (left) and velocity (right) representations for the electronic part.

bonds as compared to previous theoretical results $(1.7 \AA$ with $\triangle \mathrm{SCF}$ versus $1.6 \AA$ at the $\operatorname{CASSCF}(10,8)^{10}$ and the SSR- $\omega \mathrm{PBEh}^{13}$ levels of theory). Consequently, the lateral $\mathrm{C}-\mathrm{C}$ bond lengths at the symmetrical MECI are equally elongated by $\sim 0.1 \AA$ as compared to multireference results $(1.9 \AA$ with $\triangle \mathrm{SCF}$ versus $1.8 \AA$ and $1.7 \AA$ optimised with $\operatorname{SA}-\operatorname{CASSCF}(8,7)^{11}$ and SSR$\omega \mathrm{PBEh}^{13}$ methods respectively). At the asymmetrical MECI the lateral $\mathrm{C}-\mathrm{C}$ bond lengths are in line with the $\operatorname{SA}-\operatorname{CASSCF}(8,7)^{11}$ results $(1.4 \AA$ versus $1.5 \AA$ and $2.3 \AA$ versus $2.2 \AA$ obtained with $\triangle \mathrm{SCF}$ and $\mathrm{SA}-\mathrm{CASSCF}(8,7)$ respectively), whereas a smaller difference between both bonds was measured using the ensemble DFT method. ${ }^{13}$ The asymmetric MECI is about $0.3 \mathrm{eV}$ more stable than the symmetric MECI at the $\triangle$ SCF B3LYP/TZV2PGTH level, comparable to $0.7 \mathrm{eV}$ at the MS-MR-CASPT2/cc-pVDZ level ${ }^{11}$ and $0.09 \mathrm{eV}$ at the SSR- $\omega \mathrm{PBEh} / 6-31 \mathrm{G}^{*}$ level. ${ }^{13}$ There is no significant difference in electronic structure at these stationary points, analogous to previous results. ${ }^{13}$

After 500 propagation steps ( 250 fs of simulation time) 29 trajectories out of 250 still remained in the $S_{1}$ state. In fact, 14 trajectories never hopped to the ground state despite passing through several CIs, while the other 15 have reactivated back to $\mathrm{S}_{1}$ through the second CI shortly after hopping to the ground electronic state via the first CI, as the time between the two CI encounters was insufficient to effectively redistribute the excess electronic energy into vibrational degrees of freedom. With the exception of two trajectories, all trajectories displayed carbon monoxide ejection, which is cleaved off in the first excited state. All NAMD trajectories started with the pyramidalisation of the carbonyl $\mathrm{C}$ atom and prolongation of the $\mathrm{C}=\mathrm{O}$ bond length, a ubiquitous pattern of the $\mathrm{n} \rightarrow \pi^{*} \mathrm{C}=\mathrm{O}$ group excited state, as visualised in Fig. $2 .^{74}$

Whether the lateral C-C bond lengths stay equal or start to deviate, the trajectory takes the symmetric or the asymmetric pathway. Distinction between the two mechanisms was made on the complete evolution of lateral $\mathrm{C}-\mathrm{C}$ bond lengths. If their difference did not exceed $0.1 \AA$ while both were still shorter than $2 \AA$ then the mechanism was classified as symmetric, otherwise as asymmetric. 54 trajectories evolved initially within the first criteria, while the remaining 193 fell under the latter, giving the same ratio between symmetric and asymmetric pathways as counted by Filatov and coauthors which distinguished between the two pathways based on the difference of lateral $\mathrm{C}-\mathrm{C}$ bond lengths at the $\mathrm{CI}$ points while omitting other 


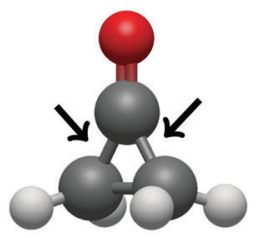

(a) $S_{0}$ minimum

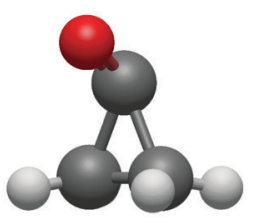

(d) $S_{1}$ minimum

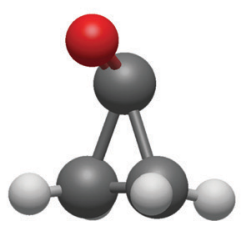

(g) symmetrical MECI

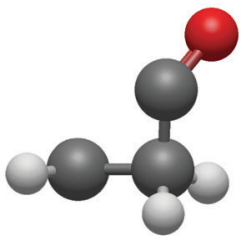

(j) asymmetrical MECI

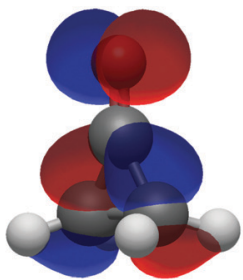

(b) $\mathrm{HOMO}$

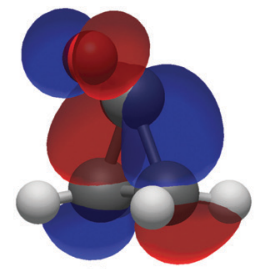

(e) HOMO

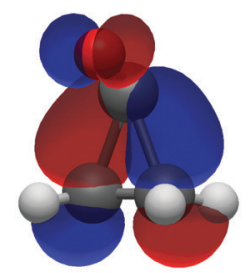

(h) HOMO

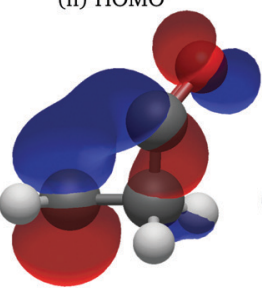

(k) HOMO

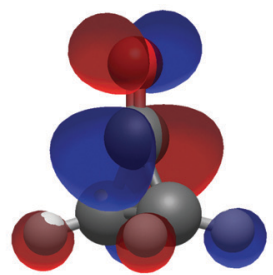

(c) LUMO
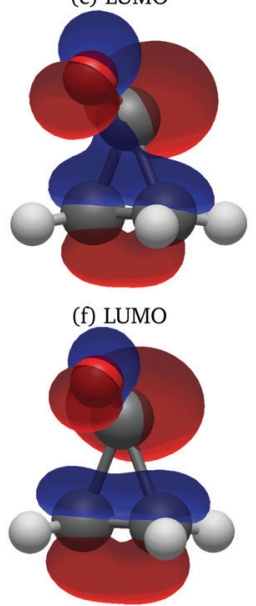

(i) LUMO

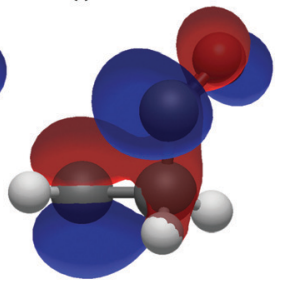

(1) LUMO
Fig. 2 Geometry, HOMO and LUMO at the $S_{0}(a-c)$ and $S_{1}(d-f)$ minima, symmetrical $(\mathrm{g}-\mathrm{i})$ and asymmetrical $\mathrm{MECl}(\mathrm{j}-\mathrm{l})$ of cyclopropanone optimised using the $\triangle S C F$ method. The arrows in subfigure (a) denote the lateral $\mathrm{C}-\mathrm{C}$ bonds, whereas the third $\mathrm{C}-\mathrm{C}$ bond is denoted distal.

Table 1 Comparison of the cyclopropanone structural parameters at the $\mathrm{S}_{0}$ minimum geometry, where $\mathrm{C}_{\mathrm{C}}$ indicates the carbonyl $\mathrm{C}$ atom

\begin{tabular}{|c|c|c|c|}
\hline Cyclopropanone & This work & Exp. ${ }^{73}$ & $\operatorname{CASSCF}(10,8)^{10}$ \\
\hline lateral $\mathrm{C}-\mathrm{C}[\AA]$ & 1.465 & 1.475 & 1.500 \\
\hline distal C-C $[\AA]$ & 1.576 & 1.575 & 1.556 \\
\hline $\mathrm{C}-\mathrm{H}[\AA]$ & 1.083 & 1.086 & - \\
\hline $\mathrm{C}-\mathrm{O}[\AA]$ & 1.201 & 1.191 & 1.184 \\
\hline $\mathrm{HCH}\left[{ }^{\circ}\right]$ & 115 & 115 & - \\
\hline $\mathrm{CCC}_{\mathrm{C}}\left[{ }^{\circ}\right]$ & 57 & 57 & - \\
\hline $\mathrm{CC}_{\mathrm{C}} \mathrm{C}\left[{ }^{\circ}\right]$ & 65 & 64 & - \\
\hline $\mathrm{CC}_{\mathrm{C}} \mathrm{O}\left[{ }^{\circ}\right]$ & 147 & - & 148 \\
\hline
\end{tabular}

details. ${ }^{13}$ We avoided such a simplified criterion, but rather used the full evolution of each system to distinguish between pathways, because the symmetric and asymmetric MECIs belong to the same CI seam, differing just slightly in energy, as discussed above. The energy evolution of exemplary NAMD trajectories undergoing symmetric and asymmetric pathways is shown in Fig. 3.
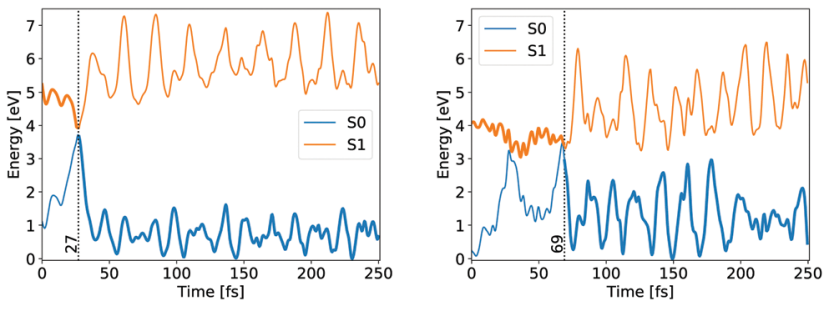

Fig. 3 Ground state (blue) and first singlet excited state (orange) energy evolution of exemplary trajectories following the symmetrical (left) and asymmetrical (right) pathways. The bold line indicates the occupied state and the vertical dashed line indicates the hop time.

In both cases the electronic ground state energy increases while the first excited state decreases, closing the gap between the two states as the system moves towards the $S_{1}$ excited state local minimum, shown in Fig. 2. The $\mathrm{S}_{1}$ minimum is $C_{\mathrm{s}}$ symmetric, with an out of cyclopropane plane positioned $\mathrm{CO}$ group lying in the reflection plane that intersects the distal $\mathrm{C}-\mathrm{C}$ bond (i.e. the $\mathrm{C}-\mathrm{C}$ bond not adjacent to the carbonyl group). The $\mathrm{C}$ atom of the CO group is displaced from ethylene by an additional $0.3 \AA$ compared to the ground state minimum structure. Any further extension brings the system to the CI between the $S_{1}$ and $S_{0}$ states, which at the $\triangle S C F$ level is just $0.1 \mathrm{eV}$ above the $\mathrm{S}_{1}$ minimum. Given that the sampled population excitation energy in its starting Franck-Condon region is on average $1.48 \mathrm{eV}$ (individual trajectories range from 0.80 to $3.24 \mathrm{eV}$ ) above the $S_{1}$ minimum energy, the CI seam with the ground electronic state is easily accessible. Trajectories exhibiting an immediate $\mathrm{SH}$ to the ground state continue distancing the $\mathrm{CO}$ group further from ethylene. In fact, the cleavage is accelerated by the repulsive nature of the ground state gradient in this configuration region. The $\mathrm{CO}$ group dissociation is mostly symmetrical with the lateral $\mathrm{C}-\mathrm{C}$ bonds extending simultaneously, and as the $\mathrm{CO}$ group has gained initial angular momentum in rotation out of the carbon atoms plane during the motion towards the $\mathrm{S}_{1}$ minimum, it continues to rotate in the initial $C_{\mathrm{s}}$ plane after splitting from ethylene. Because the system contained no initial angular momentum, the ethylene rotates in the counter direction around its newly formed double carbon bond canceling the system's total angular momentum. The ethylene double bond shows an increase in vibrational amplitude, while it diminishes in the cleaved CO. Those trajectories that overshoot the first CI and remained in the $S_{1}$ state also continue with the CO dissociation although the pattern is more asymmetric. This is because the symmetrical stretch of the CO from ethylene within the reflection plane in the $S_{1}$ state is not dissociative. In fact, the $S_{1}$ energy rises at the $\triangle \mathrm{SCF}$ level as observed from a detailed analysis of the CO dissociation by symmetrically increasing the distance between the carbon $\mathrm{CO}$ atom and the midpoint of the distal $\mathrm{C}-\mathrm{C}$ bond in the $\mathrm{S}_{1}$ minimum structure (see Fig. 4). The same trend is reproduced with state-averaged CASSCF, XMS-CASPT2 and TDDFT applying the hybrid B3LYP functional. All three methods agree on the state character change from the localised $\mathrm{CO} n \rightarrow \pi^{*}$ to a charge transfer from an ethylene localised $\pi \mathrm{MO}$ to a CO localised $\pi^{*} \mathrm{MO}$, which occurs adiabaticaly around the CI. At the CASSCF and CASPT2 


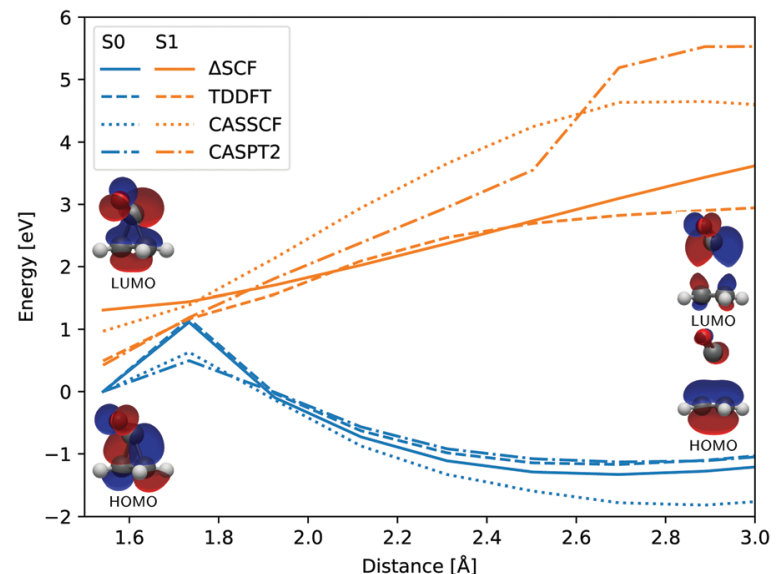

Fig. 4 Evolution of the ground state (blue) and first singlet excited state (orange) energies with respect to the distance between the center of the distal C-C bond and the carbon atom of the CO group, calculated the $\triangle \mathrm{SCF}$ (full line), TDDFT (dashed line), $\operatorname{CASSCF}(8,7)$ (dotted line) and CASPT2 $(8,7)$ (dash-dotted line) levels of theory. All electronic energies are relative to the ground state energy of the $S_{1}$ minimum structure optimised at the $\triangle \mathrm{SCF}$ level. The shown HOMO and LUMO correspond to the first and final geometries.

levels, the flattened $\mathrm{S}_{1}$ line starting from a distance of $2.7 \AA$ and $2.9 \AA$ A respectively corresponds to a $\pi \rightarrow \pi^{*}$ excitation localised on CO. At short distances, the inclusion of dynamic correlation by the CASPT2 method reduces the excitation energy as compared to the CASSCF results. A steep increase in the $\mathrm{S}_{1}$ energy calculated with the CASPT2 method between $2.5 \AA$ and $2.9 \AA$ is remarked. Whereas several orbitals in the active space at a distance of $2.5 \AA$ involve contributions from both molecules, they are located on either the carbon monoxide or the ethylene molecule at a distance of $2.9 \AA$ A.

Steered by the repulsive symmetrical $\mathrm{S}_{1} \mathrm{CO}$ dissociation part, the CO dissociation continues asymmetrically in the excited state and one of the lateral $\mathrm{C}-\mathrm{C}$ bonds breaks before the other. In this process, an excitation transfer from the $\mathrm{CO}$ to the ethylene is observed, adiabatically changing from the $\mathrm{CO}$ $n \rightarrow \pi^{*}$ excitation character to $\pi \rightarrow \pi^{*}$ of the ethylene $S_{1}$ excited state. With the electronic excitation transferred on ethylene, it starts to move towards its local $\mathrm{S}_{1}$ minimum with a profound torsion between the two $\mathrm{CH}_{2}$ groups. Because a CI with the ground electronic state resides near this minimum, a nonadiabatic transition to $\mathrm{S}_{0}$ can easily occur when the torsional angle between the two $\mathrm{CH}_{2}$ groups approaches $90^{\circ}$. If the $\mathrm{SH}$ happens, the ethylene reforms its double carbon bond and repositions its $\mathrm{CH}_{2}$ groups into their mutual plane, with extensive torsional vibrations as the system tries to reach its ground state minimum. The $\mathrm{CO}$ dissociation continues as there is no strong interaction between the two systems in the ground state. If, however, no NA transition takes place at the ethylene type $\mathrm{CI}$, the two $\mathrm{CH}_{2}$ groups continue twisting around the $\mathrm{C}-\mathrm{C}$ bond in the excited state, as being barrierless, until another ethylene type CI is reached as the ground state energy rises periodically with torsion of the $\mathrm{C}=\mathrm{C}$ bond.

A randomly selected subset of trajectories have also been propagated using the XMS-CASPT2 $(8,7)$ method. $^{70-72}$ The resulting deexcitation pathways were analogous to the observations detailed above.

\subsection{Solvated cyclopropanone}

In aqueous solution, the oxygen atom of cyclopropanone can form hydrogen bonds (H-bonds) with the surrounding water molecules. During a 45 ps long ground state DFT-MD trajectory the $\mathrm{O}_{\text {cyc. }} \cdots \mathrm{H}_{\text {wat. }}$ distance with the closest water molecule was about half the time below $2 \AA$, not becoming shorter than $1.5 \AA$ at any point (see Fig. 5). At around a quarter of the time, two hydrogen atoms of distinct water molecules are within $2 \AA$. The position of the first peak in the radial distribution function (RDF) of the $\mathrm{O}_{\text {cyc. }} \cdots \mathrm{H}_{\text {wat. }}$ distances coincides with the value of $1.95 \AA$ A determined in cyclobutanone-water and cyclohexanonewater complexes studied in the gas phase. ${ }^{75,76}$ The top lefthand-side of the first $\mathrm{O}_{\text {cyc. }} \cdots \mathrm{O}_{\text {wat. }}$. radial distribution peak at $2.85 \AA$, belonging to the oxygen atoms of water involved in $\mathrm{H}$-bonds with cyclopropanone, is similar to the corresponding distance of $2.91 \AA$ measured in cyclobutanone-water. ${ }^{75}$ The absence of any structure in the $\mathrm{H}_{\text {cyc. }} \cdots \mathrm{O}_{\text {wat. }}$ RDF curve indicates no significant water interaction with the alkane part of the ring. A more detailed spatial distribution of the location of the closest water hydrogen atoms around the carbonyl oxygen atom indicates that the majority of water molecules lay in the same plane as the cyclopropanone ring. This is in line with the position of the non-bonding electron pairs on the oxygen atom, and that the perpendicular $\pi$ orbital engages less in interaction with water molecules. The overall effect of the solvent and $\mathrm{H}$-bonds on the structure of cyclopropanone is negligible. The $\mathrm{C}=\mathrm{O}$ and the distal $\mathrm{C}-\mathrm{C}$ bond are extended by on average 0.015 and $0.007 \AA$ A respectively, while the two lateral C-C bonds are compressed by $0.011 \AA$, when compared to the corresponding minimum ground state vacuum structure. The $\mathrm{C}-\mathrm{H}$ bonds are on average longer by merely $0.002 \AA$.

The theoretical absorption spectrum of solvated cyclopropanone in the periodic water box was calculated from the 250 initial configurations sampled from the same 45 ps long MD simulation.

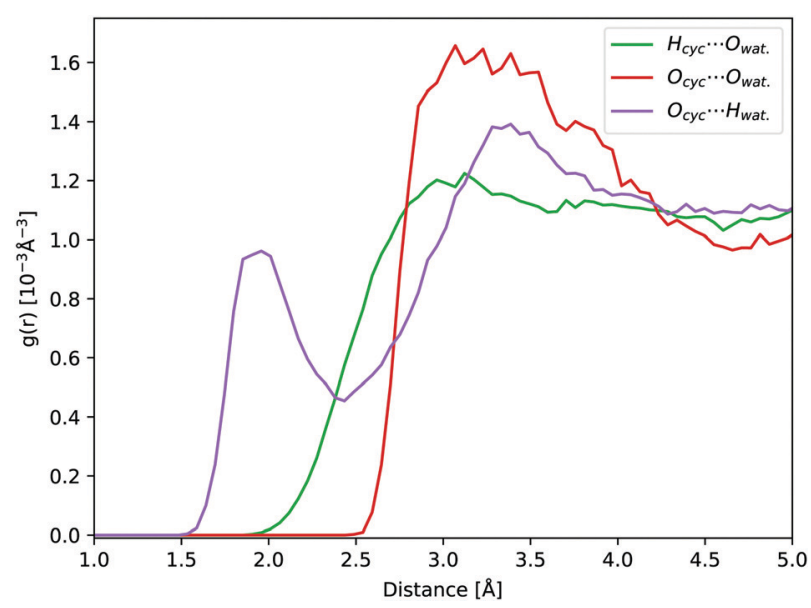

Fig. 5 RDFs obtained from a 45 ps ground state DFT-MD trajectory of cyclopropanone in aqueous solution: $O_{\text {cyc. }} \cdots O_{\text {wat. }}$ (red line), $O_{c y c} \cdots H_{\text {wat. }}$. (purple line), and $\mathrm{H}_{\mathrm{cyc}} \cdots \mathrm{O}_{\text {wat. }}$ (green line). 
The spectrum maxima in the length and velocity representation are blue-shifted by 14 and $22 \mathrm{~nm}$ respectively compared to the corresponding vacuum values of cyclopropanone (Fig. 1). The effect was traced to the $\mathrm{H}$-bonds and electrostatic interaction of the $n \rightarrow \pi^{*}$ excitation character with the water molecules that stabilises the ground state, whereas the first singlet excited state is destabilised by the decreased electron density in the oxygen $\mathrm{n}$ orbital. The aforementioned geometrical effects exerted on the cyclopropanone induce in fact a slight redshift when the vertical excitation is computed in vacuum on an average solvated cyclopropanone geometry. In the velocity representation, the prominent shoulder at around $355 \mathrm{~nm}$ observed in the gas-phase spectrum is not present in the liquid phase. In the length representation, a small shoulder can still be discerned at $335 \mathrm{~nm}$.

The encountered mechanisms resemble those in a vacuum, namely $13 \%$ and $87 \%$ of the jumps exhibit a symmetric or asymmetric character respectively, categorised based on the same criteria as the vacuum trajectories. $5 \%$ did not deactivate to the ground state at the end of the simulation time. In contrast to the gas-phase, $14 \%$ of the trajectories restore cyclopropanone after deexcitation, following either symmetrical or asymmetrical pathways. The first trajectory displaying reconnection of a broken bond hopped after 57.5 fs. In fact, none of the former trajectories deactivated at the first CI encounter. The excess energy after the hop is manifested in the vibrational motion of the cyclopropanone ring.

The H-bonds between the cyclopropanone and water molecules are disrupted in the first stage of the NAMD. As the time evolution of the $\mathrm{O}_{\text {cyc. }} \cdots \mathrm{H}_{\text {wat. }}$ RDF shown in Fig. 6 reveals, the dissociative motion of the $\mathrm{CO}$ group stretches the $\mathrm{H}$-bonds present between the oxygen atom of cyclopropanone and the hydrogen atoms of water as the CO moves from the ethylene. This process takes about 50 fs to complete, as illustrated by the disappearance of the first peak of the $\mathrm{O}_{\text {cyc. }} \cdot \mathrm{H}_{\text {wat. }}$ radial distribution density in Fig. 6 . The very first

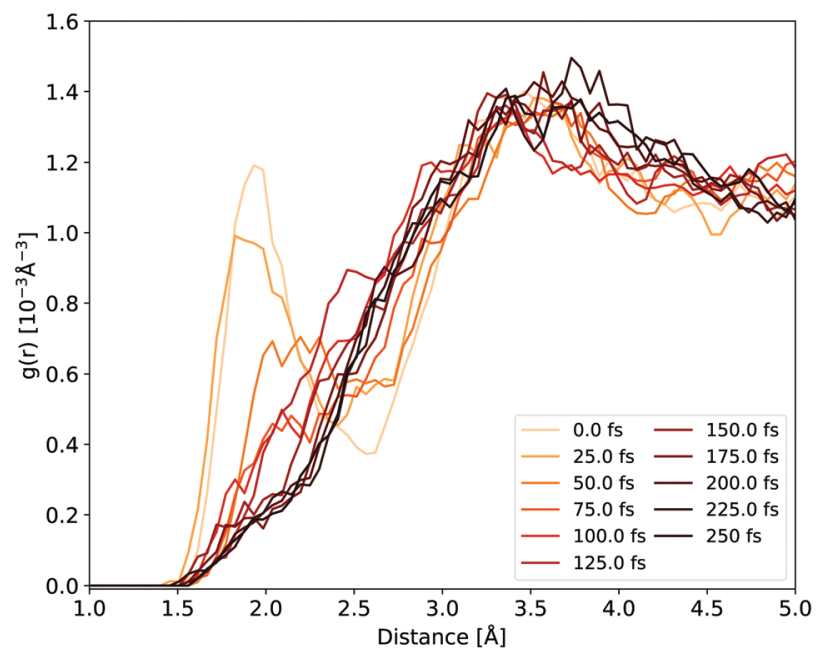

Fig. $6 \mathrm{O}_{\text {cyc. }} \cdot \mathrm{H}_{\text {wat. }}$ RDF evolution during the NAMD evolution, averaged over all solvated cyclopropanone trajectories.
Table 2 Fitting parameters and the coefficients of determination $\left(R^{2}\right)$ of the monoexponential population decay function $P_{S_{1}}(t)=\exp \left(-\left(t-t_{0}\right) / \tau\right): t \geq t_{0}$, with 1:t $<t_{0}$

\begin{tabular}{llll}
\hline Cyclopropanone & $t_{0}[\mathrm{fs}]$ & $\tau[\mathrm{fs}]$ & $R^{2}$ \\
\hline Gas & $17.4 \pm 0.5$ & $119.6 \pm 0.8$ & $98.8 \%$ \\
Solution & $8.9 \pm 0.5$ & $89.3 \pm 0.7$ & $98.5 \%$
\end{tabular}

step of this process is the carbonyl group out-of-plane movement, which disrupts some of the $\mathrm{H}$-bonds but the geometric change is still localised within its first solvation shell so the peak remains. Only when the CO starts to significantly move from the ethylene part (either by complete or partial cleavage) is a decay of the first peak observed. Since the departing CO moves away with significant kinetic energy, no H-bonds were observed reforming with the water molecules.

\subsection{Cyclopropanone $S_{1}$ lifetimes}

Filatov et al. ${ }^{13}$ fitted the $\mathrm{S}_{1}$ population decay of cyclopropanone in a vacuum with a biexponential function giving lifetimes of 33 fs and 95 fs using 100 trajectories propagated at the SSR$\omega \mathrm{PBEh} / 6-31 \mathrm{G}^{*}$ level of theory, whereby 15 non-deactivated trajectories were excluded from the analysis. Cui et al. obtained $\mathrm{S}_{1}$ lifetimes ranging from 71 fs to $126 \mathrm{fs}$ at the $\operatorname{SA}-\operatorname{CASSCF}(8,7) /$ cc-pVDZ level of theory, depending on the initial sampling conditions. ${ }^{11}$ More specifically, four sets of initial conditions were sampled in their work, each corresponding to a vibrational mode of the CO group in cyclopropanone as these modes are mostly correlated with the $S_{1}$ to $S_{0}$ transition. In our work, a monoexponential function proved accurate to fit the $S_{1}$ population decay of an isolated cyclopropanone molecule. The fitted parameters are given in Table 2 and the corresponding $S_{1}$ state decay curves are shown in Fig. 7. The cyclopropanone lifetime is longer as compared to the results of Filatov et al. ${ }^{13}$ and Cui et al., ${ }^{11}$ presumably since a larger number of trajectories were propagated, and all trajectories that reached 250 fs were included in the calculation of the lifetime and all vibrational modes were sampled. The first hop happens at $21 \mathrm{fs}$ and $17.5 \mathrm{fs}$ for cyclopropanone in a vacuum and in solution respectively. In the condensed phase, a shorter lifetime of $98 \mathrm{fs}$ is found compared to $137 \mathrm{fs}$ in a vacuum. More trajectories hop between

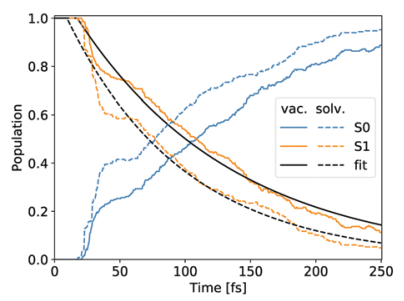

(a)

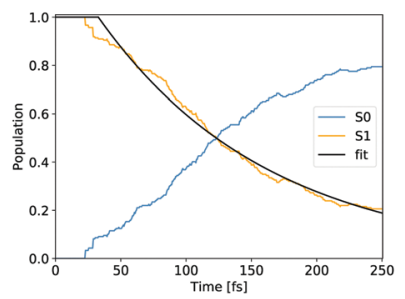

(b)
Fig. 7 Population evolution of cyclopropanone (a) and cyclopropanone hydrate (b). The blue, orange and black curves correspond to the ground state, first singlet excited state and monoexponential fit respectively. The full lines in (a) denote the vacuum, while the dashed lines indicate the aqueous environment. 
$30 \mathrm{fs}$ and $40 \mathrm{fs}$ after the start of the dynamics for cyclopropanone in the condensed phase as compared to the gas phase, all of which result in the formation of ethylene and carbon monoxide through either a symmetrical or asymmetrical pathway.

\subsection{Cyclopropanone hydrate}

In water, carbonyl functional groups are in equilibrium with a geminal diol. ${ }^{77}$ The equilibrium of the nucleophilic addition reaction of a water molecule is shifted towards the hydrate form for cyclopropanone, as the angle strain in the three-membered ring is reduced by the formation of an $\mathrm{sp}^{3}$ hybridisation of the carbonyl carbon atom. ${ }^{2,20}$ However, determination of the equilibrium constant is experimentally complicated. ${ }^{78}$ Hence, not only the photodynamics of cyclopropanone, but also of its hydrate were modelled in this work.

Compared to solvated cyclopropanone, the H-bond network between water and cyclopropanone hydrate is more versatile. In the starting conformation of the ground state DFT-MD NVT simulation, both hydrate $\mathrm{O}-\mathrm{H}$ bonds were facing the same parallel orientation giving $C_{\mathrm{s}}$ symmetry, as shown in Fig. 8 . 45 ps of NVT dynamics reveal that both $\mathrm{OH}$ groups are strongly interacting with neighbouring water molecules. The $\mathrm{H}$ atoms of cyclopropanone hydrate constantly connect to oxygen atoms of water, interrupted only when changed between water molecules. Oxygen atoms of the hydrate are also interacting with neighbouring water $\mathrm{H}$ atoms with a frequent observation of both oxygen lone pairs simultaneously bonding two neighboring water molecules. Since the $\mathrm{OH}$ groups are geminal, another observed feature is a single water molecule located close to both groups which switches the H-bond between the two hydrate oxygen atoms. As a result, the conformation of the hydroxyl groups changes minimally. Hence, the initial $C_{\mathrm{s}}$ symmetry is maintained. Prominent peaks of the corresponding radial distribution function curves (see Fig. 8) confirm the strong $\mathrm{H}$-bondings, particularly the $\mathrm{H}_{\mathrm{O} \text {,cyc. }} \cdots \mathrm{O}_{\text {wat. }}$ and $\mathrm{O}_{\text {cyc. }} \cdots \mathrm{O}_{\text {wat. }}$, where the maximum of the latter is separated by

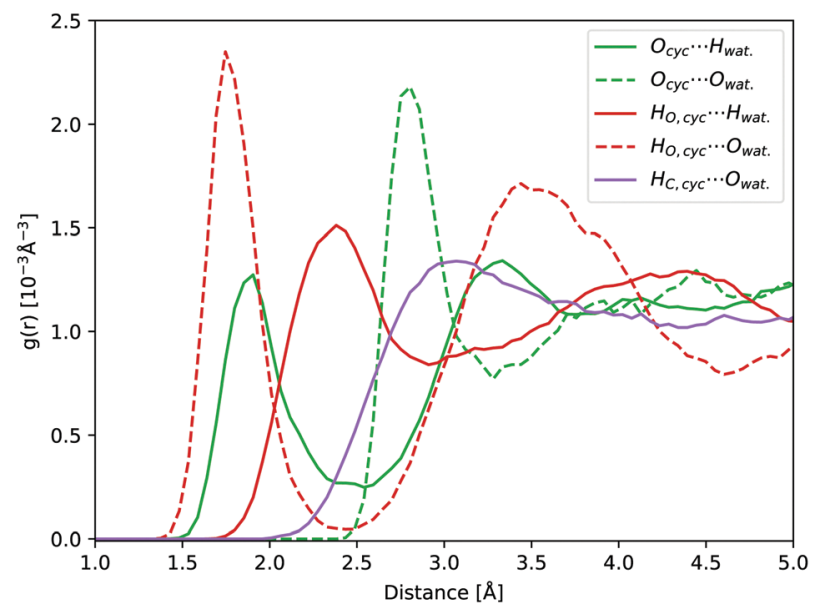

Fig. 8 Hydrate NVT RDFs: $O_{\text {cyc. }} \cdots O_{\text {wat. }}$ (green dashed line), $\mathrm{O}_{\text {cyc. }} \cdots \mathrm{H}_{\text {wat. }}$ (green line), $\mathrm{H}_{\mathrm{O}, \text { cyc. }} \cdot \mathrm{O}_{\text {wat. }}$ (red dashed line), $\mathrm{H}_{\mathrm{O}, \text { cyc. }} \cdots \mathrm{H}_{\text {wat. }}$ (red line), and $\mathrm{H}_{\mathrm{C}, \text { cyc. }} \cdots \mathrm{O}_{\text {wat. }}$ (purple line). the average $\mathrm{OH}$ water bond length from the maximum of the former curve. The height of the $\mathrm{O}_{\text {cyc. }} \cdots \mathrm{H}_{\text {wat. }}$ is almost half the size of $\mathrm{H}_{\mathrm{O} \text {,cyc. }} \cdots \mathrm{O}_{\text {wat. }}$ due to more frequent $\mathrm{H}$-bond changes between water hydrogen and hydrate oxygen atoms. Again, no significant $\mathrm{H}$-bond interaction was observed with the hydrate alkane part.

The hydrate's first excited singlet state is a transition originating from the $\sigma$ orbitals of the lateral $\mathrm{C}-\mathrm{C}$ bonds to the $\sigma^{*} \mathrm{KS}$ MO located on both hydroxyl groups, as shown in Fig. 9. In the Frank-Condon region, two higher excited states are close in energy, the former being the $\mathrm{n} \rightarrow \sigma^{*}$ located on the $\mathrm{OH}$ groups, while the latter has a similar character to $\mathrm{S}_{1}$, but involves a different $\sigma$ bonding orbital. However, in solution, preliminary analyses showed that the energy gap between these states and the $S_{1}$ state quickly increased in energy during the dynamics, making the $S_{1}$ sufficient for the NAMD simulation. In addition, calculations of the $S_{2}$ and $S_{3}$ states contained contributions from the orbitals of non-surrounding solvent molecules. The cyclopropanone hydrate absorption spectrum was calculated on the 250 sampled NAMD initial points from the NVT trajectory (see Fig. 1). The maxima are blue-shifted by 48 and $50 \mathrm{~nm}$ for the length and velocity representations respectively as compared to the corresponding maxima of solvated cyclopropanone.

Overall, $20 \%$ of the trajectories were still in the $S_{1}$ state after $250 \mathrm{fs}$ of NAMD. At the end of the simulation time, one lateral $\mathrm{C}-\mathrm{C}$ bond was broken in all trajectories which remained in the first singlet excited state and three quarters of the geminal diols lost a proton to the solvent through the Grotthuss mechanism. ${ }^{79}$ In contrast to the decay of cyclopropanone in an aqueous environment, where $3 \%$ and $87 \%$ of the trajectories decayed following the symmetrical and asymmetrical pathways respectively, all solvated cyclopropanone hydrate trajectories decayed to the ground state through an asymmetrical pathway. In the majority of the cases one of the lateral $\mathrm{C}-\mathrm{C}$ bonds was already broken (based on the $2 \AA$ criterium) at the point of the non-adiabatic hop, where only in a very small fraction both lateral $\mathrm{C}-\mathrm{C}$ bonds were still under $2 \AA$. The minimum difference between the lateral $\mathrm{C}-\mathrm{C}$ bond lengths at the jump was $\sim 0.4 \AA$, whereas the threshold distinguishing symmetrical from asymmetrical trajectories was defined in section 3.1 as $0.1 \AA$. In addition, several trajectories showed a proton transfer to the solvent, which initiated a proton exchange between water molecules via the Grotthuss mechanism ${ }^{79}$ while still in the first excited state.

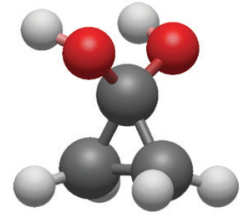

(a)

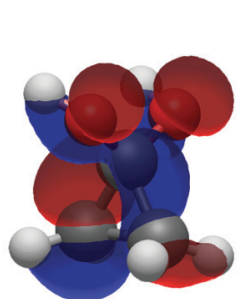

(b)

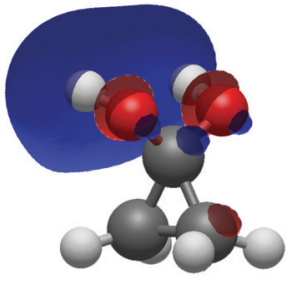

(c)
Fig. 9 Geometry (a), HOMO (b) and LUMO (c) at the $C_{s} S_{0}$ minimum of cyclopropanone hydrate optimised using the $\triangle$ SCF method. 

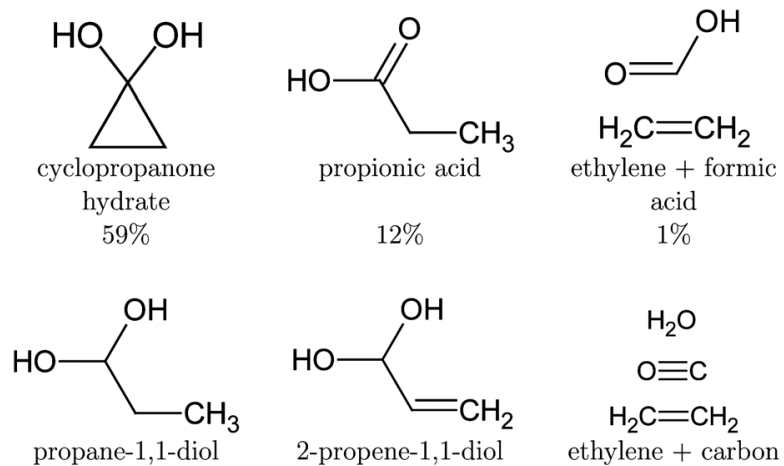

$1 \%$

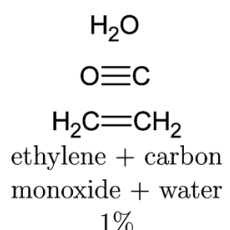

$1 \%$

Fig. 10 Photoproducts of the cyclopropanone hydrate NAMD calculations. The remaining trajectories decayed to the ground state, but the intermediate structures did not form a stable product by the end of the simulation time. Proton transfer will result in one of the above products.

After deexcitation, in $59 \%$ of the trajectories the broken lateral $\mathrm{C}-\mathrm{C}$ bond was restored by the end of the simulation time. Therefore, vibrationally hot cyclopropanone hydrate is the main product of the non-radiative deactivation mechanism, as shown in Fig. 10. In $15 \%$ of the NAMD trajectories, the second lateral $\mathrm{C}-\mathrm{C}$ bond was also broken, resulting in the formation of ethylene and a carbene fragment. The latter is stabilised by the hydrogen atoms of the surrounding water molecules. An intramolecular proton transfer from a hydroxyl group to the carbene carbon atom resulted in the formation of formic acid in one trajectory. Several other trajectories displayed a clear carbene-water interaction, however, the simulation time was too short to observe any intermolecular formation of formic acid. It is noteworthy that in one trajectory, an additional fragmentation of the carbene fragment yielded carbon monoxide, hydroxide and a solvated proton as photoproducts. Although decarboxylation is the main decomposition pathway of formic acid in aqueous solution, the dehydration is known to be catalysed by water as well. ${ }^{80,81}$ Finally, in the remaining $26 \%$ of the trajectories the lateral $\mathrm{C}-\mathrm{C}$ bonds were not restored nor broken forming a three carbon atom straight chain whereby the central methylene group relaxes to the unstrained tetrahedral structure. The second methylene group exhibits profound vibrational motion, displaying in particular scissoring and wagging modes, before pyramidalising when interaction with a solvent molecule is established. If a hydroxyl proton is expelled, the carboxyl group planarises. Remarkably, in two trajectories, an intramolecular proton transfer from the central methylene group to the carbon atom of the diol occurred, creating 2-propene-1,1diol. A hydroxyl proton was expelled at the end of the simulation time for half of the diols, mostly after the deactivation to the ground state. In most of these trajectories, the non-central methylene group extracted a proton from a nearby water molecule thereby forming propionic acid. In one case, propane-1,1-diol was created as the non-central methylene group extracted a proton from the solvent without deprotonation of a hydroxyl group. All photoproducts of the cyclopropanone hydrate NAMD calculations are summarised in Fig. 10.

The population decay of the $S_{1}$ excited state was fitted with a monoexponential function as shown in Fig. 7b. The obtained lifetime of 163 fs with a 33 fs offset and $R^{2}$ of 99.1 is slightly longer than for solvated cyclopropanone.

\section{Conclusions}

The photodissociation of cyclopropanone in a vacuum and in solution has been studied using NAMD with $\triangle$ SCF. The gasphase results are consistent with previous multireference studies, ${ }^{11,13}$ proving that $\triangle \mathrm{SCF}$ is a viable method to simulate photodecomposition processes, particularly for systems exhibiting single reference excited states. A lifetime of $137 \mathrm{fs}$ was obtained by a monoexponential fit of the $\mathrm{S}_{1}$ population decay and cyclopropanone dissociated in $99 \%$ of the NAMD trajectories. The deactivation was found to proceed through a symmetrical pathway for $22 \%$ of the molecules.

Moreover, the $\triangle \mathrm{SCF}$ method can be easily applied to the condensed phase, which has been shown with DFT using a mixed Gaussian and plane wave approach and periodic boundary conditions, also using subsystem DFT. ${ }^{30,31}$ Solvated in water, cyclopropanone is in equilibrium with cyclopropanone hydrate. Whereas the hydrate readily engages in hydrogen bonding with the surrounding solvent molecules, the oxygen atom of cyclopropanone forms hydrogen bonds only in half of the ground state DFT-MD simulation time. No significant geometrical changes of the cyclopropanone molecule are observed after solvation. The excitation of solvated cyclopropanone to the first singlet excited state results in less photolysis as compared to the gas-phase deactivation. More specifically, the decay pathway manifested a reformation of the lateral $\mathrm{C}-\mathrm{C}$ bond following a hop at either a symmetrical or asymmetrical CI in $14 \%$ of the trajectories. The deactivation mechanisms are analogous to the pathways in a vacuum. Due to the increased kinetic energy, manifested as energetic vibrational motion or dissociation, the hydrogen bonds with the solvent are disrupted during the NAMD.

The NAMD simulations of cyclopropanone hydrate revealed the cyclopropanone hydrate, propionic acid, 2-propene-1,1-diol, propane-1,1-diol and formic acid as photoproducts, in addition to conformers where one or both lateral $\mathrm{C}-\mathrm{C}$ bonds were broken, but no proton was transferred yet after $250 \mathrm{fs}$ of NAMD. All trajectories deexcited following an asymmetrical mechanism. A lifetime of 98 fs and 163 fs was obtained for cyclopropanone and cyclopropanone hydrate in an aqueous environment respectively. Finally, UV absorption spectra at a $\Delta$ SCF level were calculated using a new procedure involving an expansion of the mutually non-orthogonal states into a linear combination of singly-excited Slater determinants constructed from the ground state molecular orbitals. Compared to the experimental maximum of $312 \mathrm{~nm}$, the maxima in the length and velocity representations are red-shifted by merely $7 \mathrm{~nm}$ and $15 \mathrm{~nm}$ respectively. The spectra of cyclopropanone and its hydrate in the condensed phase are blue-shifted with respect to the gas-phase due to the favourable hydrogen bonding 
interaction in the ground state and reduced electrostatic interaction with the surrounding water molecules after excitation. In conclusion, the photodynamics of cyclopropanone and its hydrate in an aqueous environment were explored using full atomistic NAMD with $\triangle \mathrm{SCF}$, which allowed the hydrogen bonding and proton transfer after deactivation to be studied, offering the first insights into their condensed phase photochemistry.

\section{Conflicts of interest}

There are no conflicts to declare.

\section{Acknowledgements}

The work has been supported by the University of Zurich and the Swiss National Science Foundation (grant no. PP00P2 170667). We thank the Swiss National Supercomputing Center for the computing resources (project IDs: s875, s1001, and s1036).

\section{Notes and references}

1 N. De Kimpe, in Cyclopropanone, American Cancer Society, 2001.

2 V. P. Lipp, J. Buchkremer and H. Seeles, Justus Liebigs Ann. Chem., 1932, 499, 1-25.

3 A. D. Semenow, E. F. Cox and J. D. Roberts, J. Am. Chem. Soc., 1956, 78, 3221-3223.

4 W. B. DeMore, H. O. Pritchard and N. Davidson, J. Am. Chem. Soc., 1959, 81, 5874-5879.

5 B. J. G. Burr and M. J. S. Dewar, J. Chem. Soc., 1954, 1201-1203.

6 N. J. Turro, W. B. Hammond, P. A. Leermakers and N. J. Turro, Recl. Trav. Chim. Pays-Bas, 1966, 387, 70.

7 S. E. Schaafsma, H. Steinberg and T. J. D. Boer, Recl. Trav. Chim. Pays-Bas, 1966, 85, 1170-1172.

8 M. J. Abplanalp, A. Borsuk, B. M. Jones and R. I. Kaiser, Astrophys. J., 2015, 814, 45.

9 H. J. Rodriguez, J.-C. Chang and T. F. Thomas, J. Am. Chem. Soc., 1976, 98, 2027-2034.

10 G. Cui, Y. Ai and W. Fang, J. Phys. Chem. A, 2010, 114, 730-734.

11 G. Cui and W. Fang, J. Phys. Chem. A, 2011, 115, 1547-1555.

12 T. F. Thomas and H. J. Rodriguez, J. Am. Chem. Soc., 1971, 93, 5918-5920.

13 M. Filatov, S. K. Min and C. H. Choi, Phys. Chem. Chem. Phys., 2019, 21, 2489-2498.

14 J. Suchan, J. Janoš and P. Slavíček, J. Chem. Theory Comput., 2020, 16, 5809-5820.

15 L. M. Ibele, Y. Lassmann, T. J. Martínez and B. F. Curchod, J. Chem. Phys., 2021, 154, 104110.

16 N. J. Turro and W. B. Hammond, Tetrahedron, 1968, 24, 6017-6028.

17 N. J. Turro and W. B. Hammond, Tetrahedron, 1968, 24, 6029-6035.
18 N. J. Turro, Acc. Chem. Res., 1969, 2, 25-32.

19 B. S. E. Schaafsma, H. Steinberg and T. J. D. Boer, Recl. Trav. Chim. Pays-Bas, 1967, 86, 1170.

20 K. B. Wiberg, K. M. Morgan and H. Maltz, J. Am. Chem. Soc., 1994, 116, 11067-11077.

21 T. H. Cromartie, Biochem. Biophys. Res. Commun., 1982, 105, 785-790.

22 J. S. Wiseman and R. H. Abeles, Biochemistry, 1979, 18, 427-435.

23 J. S. Wiseman, J. S. Nichols and M. X. Kolpak, J. Biol. Chem., 1982, 257, 6328-6332.

24 K. W. Schmid, A. Hittmair, H. Schmidhammer and B. Jasani, J. Histochem. Cytochem., 1989, 37, 473-477.

25 H. H. Wasserman, G. M. Clark and P. C. Turley, Stereochemistry I, Springer, Berlin, Heidelberg, 1974, pp. 73-156.

26 I. Tavernelli, Acc. Chem. Res., 2015, 48, 792-800.

27 M. De Vetta, M. F. Menger, J. J. Nogueira and L. González, J. Phys. Chem. B, 2018, 122, 2975-2984.

28 F. Santoro, J. A. Green, L. Martinez-Fernandez, J. Cerezo and R. Improta, Phys. Chem. Chem. Phys., 2021, 23, 8181-8199.

29 K. Sneskov, T. Schwabe, O. Christiansen and J. Kongsted, Phys. Chem. Chem. Phys., 2011, 13, 18551-18560.

30 M. Mališ and S. Luber, J. Chem. Theory Comput., 2020, 16, 4071-4086.

31 M. Mališ and S. Luber, J. Chem. Theory Comput., 2021, 17, 1653-1661.

32 R. Crespo-Otero and M. Barbatti, Chem. Rev., 2018, 118, 7026-7068.

33 T. R. Nelson, A. J. White, J. A. Bjorgaard, A. E. Sifain, Y. Zhang, B. Nebgen, S. Fernandez-Alberti, D. Mozyrsky, A. E. Roitberg and S. Tretiak, Chem. Rev., 2020, 120, 2215-2287.

34 E. Tapavicza, G. D. Bellchambers, J. C. Vincent and F. Furche, Phys. Chem. Chem. Phys., 2013, 15, 18336-18348.

35 J. C. Tully, J. Chem. Phys., 1990, 93, 1061-1071.

36 G. Granucci and M. Persico, J. Chem. Phys., 2007, 126, 134114.

37 B. F. Curchod, U. Rothlisberger and I. Tavernelli, ChemPhysChem, 2013, 14, 1314-1340.

38 T. Ziegler, A. Rauk and E. J. Baerends, Theor. Chim. Acta, 1977, 43, 261-271.

39 R. O. Jones and O. Gunnarsson, Rev. Mod. Phys., 1989, 61, 689-746.

40 A. Hellman, B. Razaznejad and B. Lundqvist, J. Chem. Phys., 2004, 120, 4593-4602.

41 J. Gavnholt, T. Olsen, M. Engelund and J. Schiøtz, Phys. Rev. B: Condens. Matter Mater. Phys., 2008, 78, 075441.

42 T. Ziegler, M. Seth, M. Krykunov, J. Autschbach and F. Wang, J. Chem. Phys., 2009, 130, 154102.

43 R. J. Maurer and K. Reuter, J. Chem. Phys., 2013, 139, 014708.

44 E. Pradhan, K. Sato and A. V. Akimov, J. Condens. Matter Phys., 2018, 30, 484002.

45 G. Levi, A. V. Ivanov and H. Jónsson, J. Chem. Theory Comput., 2020, 6968-6982.

46 J. Behler, K. Reuter and M. Scheffler, Phys. Rev. B: Condens. Matter Mater. Phys., 2008, 77, 115421. 
47 G. Levi, M. Pápai, N. E. Henriksen, A. O. Dohn and K. B. Møller, J. Phys. Chem. C, 2018, 122, 7100-7119.

48 T. Kowalczyk, S. R. Yost and T. V. Voorhis, J. Chem. Phys., 2011, 134, 1-8.

49 K. Yang, R. Peverati, D. G. Truhlar and R. Valero, J. Chem. Phys., 2011, 135, 044118.

50 S. B. Worster, O. Feighan and F. R. Manby, J. Chem. Phys., 2021, 154, 124106.

51 T. Baruah, M. Olguin and R. R. Zope, J. Chem. Phys., 2012, 137, 084316.

52 D. Hait and M. Head-Gordon, J. Phys. Chem. Lett., 2021, 12, 4517-4529.

53 M. Mališ, E. Vandaele and S. Luber, Manuscript submitted for publication.

54 M. Sapunar, T. Piteša, D. Davidović and N. Došlić, J. Chem. Theory Comput., 2019, 15, 3461-3469.

55 CP2K Program Package, CP2K Developers Group, Version 6.1, https://www.cp2k.org/.

56 S. Hammes-Schiffer and J. C. Tully, J. Chem. Phys., 1994, 101, 4657-4667.

57 M. Hillery, R. F. O’Connell, M. O. Scully and E. P. Wigner, Distribution functions in physics: Fundamentals, 1984.

58 J. P. Zobel, J. J. Nogueira and L. González, Phys. Chem. Chem. Phys., 2019, 21, 13906-13915.

59 M. Krack, Theor. Chem. Acc., 2005, 114, 145-152.

60 S. Grimme, J. Antony, S. Ehrlich and H. Krieg, J. Chem. Phys., 2010, 132, 154104.

61 J. Schmidt, J. Vandevondele, I. F. W. Kuo, D. Sebastiani, J. I. Siepmann, J. Hutter and C. J. Mundy, J. Phys. Chem. B, 2009, 113, 11959-11964.

62 J. VandeVondele, F. Mohamed, M. Krack, J. Hutter, M. Sprik and M. Parrinello, J. Chem. Phys., 2005, 122, 014515.

63 T. D. Kühne, M. Iannuzzi, M. Del Ben, V. V. Rybkin, P. Seewald, F. Stein, T. Laino, R. Z. Khaliullin, O. Schütt, F. Schiffmann, D. Golze, J. Wilhelm, S. Chulkov, M. H. BaniHashemian, V. Weber, U. Borštnik, M. Taillefumier, A. S. Jakobovits, A. Lazzaro, H. Pabst, T. Müller, R. Schade, M. Guidon, S. Andermatt, N. Holmberg, G. K. Schenter, A. Hehn, A. Bussy, F. Belleflamme, G. Tabacchi, A. Glöß, M. Lass, I. Bethune, C. J. Mundy, C. Plessl, M. Watkins, J. VandeVondele, M. Krack and J. Hutter, J. Chem. Phys., 2020, 152, 194103.

64 B. G. Levine, J. D. Coe and T. J. Martínez, J. Phys. Chem. B, 2008, 112, 405-413.

65 TURBOMOLE V6.6 2014, a development of University of Karlsruhe and Forschungszentrum Karlsruhe GmbH, 19892007, TURBOMOLE GmbH, since 2007, available from http:// www.turbomole.com.
66 M. J. Frisch, G. W. Trucks, H. B. Schlegel, G. E. Scuseria, M. A. Robb, J. R. Cheeseman, G. Scalmani, V. Barone, G. A. Petersson, H. Nakatsuji, X. Li, M. Caricato, A. V. Marenich, J. Bloino, B. G. Janesko, R. Gomperts, B. Mennucci, H. P. Hratchian, J. V. Ortiz, A. F. Izmaylov, J. L. Sonnenberg, D. Williams-Young, F. Ding, F. Lipparini, F. Egidi, J. Goings, B. Peng, A. Petrone, T. Henderson, D. Ranasinghe, V. G. Zakrzewski, J. Gao, N. Rega, G. Zheng, W. Liang, M. Hada, M. Ehara, K. Toyota, R. Fukuda, J. Hasegawa, M. Ishida, T. Nakajima, Y. Honda, O. Kitao, H. Nakai, T. Vreven, K. Throssell, J. A. Montgomery, Jr., J. E. Peralta, F. Ogliaro, M. J. Bearpark, J. J. Heyd, E. N. Brothers, K. N. Kudin, V. N. Staroverov, T. A. Keith, R. Kobayashi, J. Normand, K. Raghavachari, A. P. Rendell, J. C. Burant, S. S. Iyengar, J. Tomasi, M. Cossi, J. M. Millam, M. Klene, C. Adamo, R. Cammi, J. W. Ochterski, R. L. Martin, K. Morokuma, O. Farkas, J. B. Foresman and D. J. Fox, GaussianË 16 Revision C.01, Gaussian Inc., Wallingford CT, 2016.

67 BAGEL, Brilliantly Advanced General Electronic-structure Library., http://www.nubakery.org under the GNU General Public License.

68 F. Weigend and R. Ahlrichs, Phys. Chem. Chem. Phys., 2005, 7, 3297-3305.

69 A. Schäfer, H. Horn and R. Ahlrichs, J. Chem. Phys., 1992, 97, 2571-2577.

70 B. Vlaisavljevich and T. Shiozaki, J. Chem. Theory Comput., 2016, 12, 3781-3787.

71 J. W. Park and T. Shiozaki, J. Chem. Theory Comput., 2017, 13, 2561-2570.

72 T. Shiozaki, Wiley Interdiscip. Rev.: Comput. Mol. Sci., 2018, 8, 1-7.

73 J. M. Pochan, J. E. Baldwin and W. H. Flygare, J. Am. Chem. Soc., 1968, 90, 1072-1073.

74 D. W. Liao, A. M. Mebel, M. Hayashi, Y. J. Shiu, Y. T. Chen and S. H. Lin, J. Chem. Phys., 1999, 111, 205-215.

75 S. Melandri, A. Maris, B. M. Giuliano and W. Caminati, J. Chem. Phys., 2005, 123, 164304.

76 J. Gao, N. A. Seifert and W. Jäger, Phys. Chem. Chem. Phys., 2019, 21, 12872-12880.

77 H.-J. Buschmann, H.-H. Füldner and W. Knoche, BunsenGes. Phys. Chem., Ber., 1980, 84, 41-44.

78 R. Gómez-Bombarelli, M. González-Pérez, M. T. Pérez-Prior, E. Calle and J. Casado, J. Phys. Chem. A, 2009, 113, 11423-11428.

79 N. Agmon, Chem. Phys. Lett., 1995, 244, 456-462.

80 J. Yu and P. E. Savage, Ind. Eng. Chem. Res., 1998, 37, 2-10. 81 N. Akiya and P. E. Savage, AIChE J., 1998, 44, 405-415. 